Renteneintrittsalter

\section{Gute Voraussetzungen schaffen!}

Ab 2031 gilt das gesetzliche Renteneintrittsalter von 67 Jahren. Soll die kommende Legislaturperiode unter dem Signum der Nachhaltigkeit stehen, müssen wirksame Strategien gefunden werden, damit in allen Betrieben bis zum Renteneintrittsalter gut gearbeitet werden kann - bevor über eine weitere Anhebung diskutiert wird. Tatsächlich ist in Deutschland seit zwei Jahrzehnten eine stark steigende Erwerbsbeteiligung Älterer zu verzeichnen. Offenbar braucht die Wirtschaft die älteren Arbeitnehmer:innen. Dennoch: Die Mehrheit der älteren Beschäftigten geht nach wie vor deutlich vor dem offiziellen Rentenalter in den Ruhestand. 2019 lag das tatsächliche Rentenzugangsalter bei durchschnittlich 62,3 Jahren, mehr als drei Jahre vor der Altersgrenze für die Regelaltersrente. Zudem arbeiten längst nicht alle Erwerbstätigen bis zu ihrer Altersrente: Krankschreibung, Arbeitslosigkeit und die Freistellungsphase der Altersteilzeit dienen als Brücke in die Rente. Außerdem werden Ältere von den Betrieben seltener eingestellt als Jüngere. Vor allem bei Arbeitsplatzverlust fällt es schwer, eine (adäquate) Neubeschäftigung zu finden.

Um Beschäftigte länger im Erwerbsleben zu halten, aber auch angesichts teilweise rasanter technischer und organisatorischer Neuerungen, kommt der Weiterbildung eine herausragende Bedeutung zu. Denn stetige Qualifizierung erhöht die Attraktivität von Beschäftigten. Jedoch sind nach wie vor große Defizite bei der Einbeziehung der älteren Erwerbstätigen in die betriebliche Weiterbildung zu beobachten. Während sich von 2001 bis 2017 der Anteil der Betriebe, die mindestens einem Beschäftigten die Kosten der Teilnahme an einer Weiterbildung finanzieren, von $18 \%$ auf $35 \%$ fast verdoppelt hat, verharrt der Anteil der Betriebe, die auch älteren Beschäftigten ein Weiterbildungsangebot unterbreiten, bei $7 \%$.

Viele Beschäftigte können sich aufgrund ihrer gesundheitlichen Situation nicht vorstellen, ihre Tätigkeit bis zum offiziellen Renteneintrittsalter auszuüben. Körperliche und geistige Erschöpfung tragen gleichermaßen hierzu bei. Mehr noch: Psychische Erkrankungen sind seit einigen Jahren der häufigste Grund für eine Erwerbsminderungsrente. Deshalb sollte eine alterns- und altersgerechte Arbeitsgestaltung neben physischen Aspekten auch psychischen Belastungen, wie anhaltend hohem Termin- und Leis-

(C) Der/die Autor:in 2021. Open Access: Dieser Artikel wird unter der Creative Commons Namensnennung 4.0 International Lizenz veröffentlicht (creativecommons.org/licenses/by/4.0/deed.de).

Open Access wird durch die ZBW - Leibniz-Informationszentrum Wirtschaft gefördert. tungsdruck oder langen Arbeitszeiten und Überstunden, entgegenwirken und ausreichende Erholung ermöglichen.

Etwa ein Drittel der Babyboomer berichtet von schlechten Arbeitsbedingungen, unabhängig davon, ob sie an manuellen oder nicht manuellen Arbeitsplätzen beschäftigt sind. Ein Sachverhalt, der bislang nur wenig Aufmerksamkeit erhält - und wenn, dann nur punktuell, wie in Pflegeeinrichtungen. Ob die Arbeit als gut oder schlecht empfunden wird, hängt weniger von der Branche, sondern mehr vom Betrieb, der Gestaltung der Arbeit und speziell von den Führungskräften ab. Gut gestaltete Arbeitsplätze sind gesundheitsförderlich und tragen zur Lebensqualität der Beschäftigten bei. Dabei geht es um mehr als nur Arbeitsschutz. Wer in der Arbeit Sinn und Anerkennung erfährt, Handlungsspielraum bei der Ausführung der eigenen Tätigkeit hat sowie sozialen Rückhalt erlebt, denkt seltener über einen frühen Erwerbsausstieg nach.

Im Gegensatz zu den skandinavischen Ländern herrscht in Deutschland nach wie vor eine weit verbreitete Orientierung auf den Frühausstieg - bei Beschäftigten und Personalverantwortlichen. Mehr als die Hälfte aller erwerbstätigen Babyboomer wünscht sich, so früh wie möglich aus dem Erwerbsleben auszusteigen. Das lässt sich mit schlechter Gesundheit und schlechten Arbeitsbedingungen erklären, aber längst nicht nur. Selbst wenn sowohl die eigene Arbeit und Gesundheit als gut empfunden werden, will nur ein Achtel bis zur Regelaltersgrenze erwerbstätig bleiben. Hier kommt den Unternehmensleitungen wie auch den unmittelbaren Führungskräften eine besondere Bedeutung zu. Denn diese können nicht nur Beteiligungsoptionen schaffen, sondern auch Arbeitskulturen in Unternehmen verändern. Wünschenswert wäre zudem eine Diskussion in der Gesellschaft über die Bedeutung, die Arbeit für uns hat - die Erfahrungen aus der CoronaPandemie können hier einige Impulse geben.

Mit Blick auf die Beschäftigung bis zum 67. Lebensjahr - und gegebenenfalls darüber hinaus - bedeutet Nachhaltigkeit, also eine langfristige, an der Erwerbsbiografie orientierte Beschäftigungspolitik, den Abbau von Vermittlungshemmnissen und Weiterbildungsbarrieren, eine alternsgerechte Arbeitsgestaltung, Prävention und Gesundheitsmanagement sowie eine zwischen Leistung und Well-Being ausbalancierende Arbeitskultur. Ohne abgestimmte und koordinierte Maßnahmen der verschiedenen Sozialversicherungsträger ist das nicht zu erreichen.

Götz Richter, Lutz Bellmann, Anita Tisch, Hans Martin Hasselhorn Bundesanstalt für Arbeitsschutz und Arbeitsmedizin, Institut für Arbeitsmarkt- und Berufsforschung,

Bergische Universität Wuppertal richter.goetz@baua.bund.de 